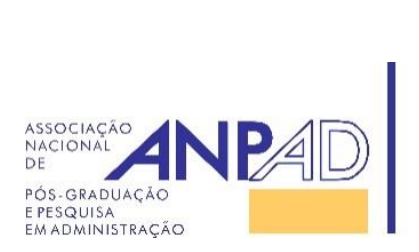

Disponível em

http://www.anpad.org.br/rac

RAC, Rio de Janeiro, v. 21, n. 5, art. 6,

pp. 710-729, Setembro/Outubro, 2017

http://dx.doi.org/10.1590/1982-7849rac2017160316

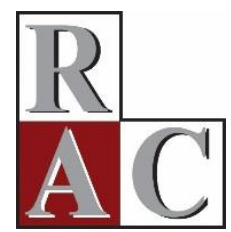

$(c)$ EY

\title{
Inventário de Suporte Organizacional à Aprendizagem: Desenvolvimento e Evidências de Validação
}

Organizational Learning Support Inventory: Development and Validation of Evidence

Bruno Chaves Correia-Lima ${ }^{1}$ Elisabeth Loiola ${ }^{1}$ Cícero Roberto Pereira ${ }^{2}$ Sônia Maria Guedes Gondim ${ }^{1}$

Universidade Federal da Bahia ${ }^{1}$ Universidade Federal da Paraíba ${ }^{2}$

Artigo recebido em 31.10.2016. Última versão recebida em 14.03.2017. Aprovado em 25.03.2017. Publicado online em 20.06.2017. 


\title{
Resumo
}

Desenvolveu-se, testou-se e validou-se um inventário (ISOA) composto pela Escala de Suportes Organizacionais à Aquisição de Competências (ESOAC) e pela Escala de Suportes Organizacionais à Transferência para o Trabalho (ESOTT). Subdivididos em material e psicossocial, suportes correspondem ao apoio organizacional para a aprendizagem individual. A partir de revisão de escalas de suportes nacionais e internacionais, elaborou-se o inventário, submetendo-o à validação de seu conteúdo (análise de juízes e pré-testes). Para a validação de construto foram aplicadas análises fatoriais exploratórias (Estudo 1) e confirmatórias (Estudo 2) a resultados coletados em um banco público e em uma Organização Não Governamental, respectivamente. O Estudo 1 permitiu propor escalas com parâmetros adequados de validade e confiabilidade para medir os constructos. O Estudo 2 confirma, por Análises Fatoriais Confirmatórias, a adequação das escalas. Contribui-se para a literatura sobre aprendizagem nas organizações na medida em que são apresentados instrumentos com evidências de validade e confiabilidade dos suportes à aprendizagem e à transferência, diferenciados porque relacionam os suportes recebidos a experiências específicas (não genéricas) e interrelacionadas de aprendizagem (aquisição/retenção) e a transferências dessas aprendizagens para o trabalho.

Palavras-chave: suporte à aprendizagem; suporte à aquisição; suporte à transferência; aprendizagem individual; escala.

\begin{abstract}
We developed, tested and validated an inventory (ISOA) composed of Organizational Support Scale for Competences Acquisition (OSSCA) and Organizational Support Scale for Transfer to Work (OSSTW). Subdivided material and psychosocial supports correspond to organizational support for individual learning. Based on a review of national and international support scales, we elaborated an inventory and submitted it to content validation (analysis by judges and pretesting). For construct validation we applied exploratory (Study 1) and confirmatory (Study 2) factor analysis to the results collected in a public bank and a non-governmental organization, respectively. Study 1 allowed proposal of scales with appropriate parameters for measuring construct validity and reliability. Study 2 confirmed scale adequacy by confirmatory factorial analysis. This study contributes to the literature on organizational learning by presenting instruments with evidence for validity and reliability of the supports to learning and transfer, differentiated by relating received support to specific experiences (non-generic) and interrelated learning (acquisition / retention) and the transfer of this knowledge to work.
\end{abstract}

Key words: learning support; acquisition support; transfer support; individual learning; scale. 


\section{Introdução}

Suportes organizacionais correspondem a práticas e mecanismos organizacionais que refletem a valorização e a preocupação das organizações em relação a seus trabalhadores (Eisenberger, Huntington, Hutchison, \& Sowa, 1986; Salehzadeh, Asadi, Pool, Ansari, \& Haroni, 2014). Pesquisa-se sua natureza e suas características motivacionais, assim como o seu papel, de forma isolada ou em relação, por exemplo, com a aprendizagem nas organizações (Perrot et al., 2014; Salehzadeh et al., 2014).

Evidências na literatura sugerem que ambientes organizacionais promotores de suporte material e psicossocial aos trabalhadores contribuem para o alcance de objetivos organizacionais (Chiaburu, Dam, \& Hutchins, 2010; Choi \& Jacobs, 2011; Coelho, Abbad, \& Todeschini, 2005; Jansen, Vera, \& Crossan, 2009).

O suporte organizacional à aprendizagem, em especial, corresponde a estímulos materiais e psicossociais oferecidos pela organização para favorecer a aprendizagem de indivíduos no trabalho (Balarin, Zerbini, \& Martins, 2014) e alcançar suas etapas de aquisição, de retenção, de generalização e de transferência. A aquisição, fase inicial do processo de aprendizagem, corresponde à apreensão de competências na memória de curto prazo, enquanto a retenção da aprendizagem é temporária, dependendo do significado que os conteúdos aprendidos têm para o indivíduo. Já a etapa de generalização compreende a aplicação de aprendizagens a contextos similares aos de aprendizagem (Mortimer \& Scott, 2002; Vygotsky, 1984), e a de transferência de aprendizagem representa a aplicação no trabalho do que foi aprendido pelos indivíduos (Blume, Ford, Baldwin, \& Huang, 2010).

A aquisição de competências e a transferência das competências aprendidas pelos indivíduos para o trabalho são fenômenos distintos que vêm sendo pesquisados por grupos diferenciados de estudiosos, com amparo em teorias e microteorias relacionadas, mas específicas, e na utilização de medidas diferenciadas. Abordagens cognitivistas preconizam que a capacidade de o aprendiz organizar os CHA'S (conhecimentos, habilidades e atitudes) aprendidos, o que facilita sua recuperação e emprego em novas situações de trabalho, é tão importante para a transferência quanto as similaridades ou diferenças entre os comportamentos antes e depois, valorizados para medir a transferência pelas abordagens pioneiras (Pantoja \& Borges-Andrade, 2004).

Amparado em tradição cognitivista, o modelo reconfigurado de transferência de aprendizagem de Holton e Baldwin (2003) divide o processo de aprendizagem em duas fases, que representam os pontos extremos de um mesmo continuum. A primeira fase inclui a aquisição de conhecimento (Know that) para ser aplicado (Know how) e promover melhorias de desempenho pela prática, ao passo que a segunda fase abarca a aplicação do conhecimento no contexto de trabalho, a repetição e a manutenção das transferências, assim como a sua aplicação a variados contextos de trabalho ao longo da vida do aprendiz. Argumenta-se que cada uma das fases de aprendizagem possui características particulares, o que sugere que exigem estímulos diferentes para que ocorram.

Escalas de suportes validadas no campo da aprendizagem individual (Coelho et al., 2005; Kraimer, Seibert, Wayne, Liden, \& Bravo, 2011; Tracey \& Tews, 2005) abordam, em regra, somente uma das etapas do processo de aprendizagem. Em vista disso, não permitem identificar se há diferenças quanto aos suportes por fase do processo de aprendizagem. Instrumentos de suportes à transferência, em especial, não permitem medir essa transferência, pressupondo sua ocorrência (Kirwan \& Birchall, 2006). Ademais, para medir transferências de aprendizagem individual para o trabalho é preciso focar o desempenho do aprendiz em contextos diferentes dos de aprendizagem (Pantoja \& Borges-Andrade, 2004), relacionando esses desempenhos ao que foi aprendido. Outra questão ainda não plenamente respondida pelas escalas de suporte revisadas é a de como avaliar adequadamente o suporte que a organização dá à aquisição de CHA's e à transferência desses CHA's aprendidos pelos indivíduos para práticas organizacionais (Blume et al., 2010).

Em meio a tantas lacunas de conhecimento, verifica-se uma crescente preocupação com a transferência de aprendizagens para o trabalho, seja porque as organizações esperam melhoria 
continuada do desempenho de seus trabalhadores, seja porque os investimentos alocados em aprendizagem tendem a ser avaliados como qualquer outro, com base nos retornos financeiros e não financeiros para as organizações, seja ainda porque os resultados de pesquisas sobre transferência de aprendizagem podem ajudar as organizações a alinhar as oportunidades de desenvolvimento de CHA's oferecidas aos seus trabalhadores a suas necessidades estratégicas (Pantoja \& Borges-Andrade, 2004).

Buscando contribuir para a área de avaliação da aprendizagem individual (aquisição e transferência de competências) no trabalho, objetiva-se desenvolver, testar e validar o Inventário de Suporte Organizacional à Aprendizagem (ISOA), composto pela Escala de Suportes Organizacionais à Aquisição de Competências (ESOAC) e pela Escala de Suportes Organizacionais à Transferência para o Trabalho (ESOTT).

\section{Suportes Organizacionais}

Quando se sentem apoiados, os funcionários mostram-se mais seguros como aprendizes e, portanto, assumem posturas pró-aprendizagem (Bauer \& Green, 1998; Perrot et al., 2014). Cultura de aprendizagem, incentivos organizacionais, disponibilização de ferramentas, de recursos materiais, de recompensas financeiras (Choi \& Jacobs, 2011; Ellinger, 2005; Lee et al., 2004), de tempo, relações com colegas de trabalho (Cheng, Wang, Yang, \& Peng, 2011; Lohman, 2005), apoio dos chefes (Clarke, 2004; Cohen, 1990), feedback à busca de aprendizagem, auxílio em dúvidas (Coelho et al., 2005), tolerância a erros (Catino \& Patriota, 2013) são alguns aspectos contextuais relacionados à aquisição de novas competências e à percepção de utilidade de novas aprendizagem por parte dos indivíduos (Choi \& Jacobs, 2011; Cohen, 1990) que se associam, por sua vez, a novos comportamentos no trabalho e a novas maneiras de executar antigas tarefas.

Múltiplos autores (Dawley, Andrews, \& Bucklew, 2008; Eisenberger et al., 1986) desenvolveram e validaram escalas que medem suportes organizacionais para promover bem-estar dos empregados e condições adequadas de trabalho, por exemplo, sem foco específico sobre aprendizagem. Essas escalas foram usadas em alguns estudos (Baranik, Roling, \& Eby, 2010; Chuo, Tsai, Lan, \& Tsai, 2011; Perrot et al., 2014; Salehzadeh et al., 2014) que objetivaram investigar a relação entre suportes organizacionais e aprendizagem nas organizações. Nesses estudos, o constructo aprendizagem foi medido por indicadores quantitativos ou por escalas preexistentes de aprendizagem. Por exemplo, Brandão, BorgesAndrade e Guimarães (2012) medem aprendizagem em termos de horas de treinamento, enquanto Baranik, Roling e Eby (2010) usam a escala de Ragins e McFarlin's (1990) de aprendizagem por mentoria/coaching. Já Chuo, Tsai, Lan e Tsai (2011) usam uma escala de intenção de uso de e-learning, e Salehzadeh et al. (2014) utilizam a Dimension of the Learning Organization (DLOQ) de Marsick e Watkins (2003).

Objetivando propor instrumentos que possibilitem medir com mais acurácia os tipos de suportes em relação às etapas de aquisição e de transferência do processo de aprendizagem, este trabalho explora a distinção entre suportes à aquisição (aprendizagem) de competências e suportes à aplicação no trabalho das competências aprendidas (transferência de aprendizagem). Embora possam ser genericamente denominados de Suportes Organizacionais, argumenta-se que cada um desses suportes se refere a fenômenos diferentes.

\section{Suportes organizacionais à aquisição de competências}

A diversificação de estímulos ao aprendiz, com foco nos processos cognitivos de atenção e de percepção, e a contínua motivação à aprendizagem podem ser estimuladas por suportes à aquisição de conhecimento (Coelho \& Mourão, 2011). Embora pesquisas sobre fatores que facilitam ou dificultam aprendizagens dos indivíduos sejam frequentes no campo da aprendizagem individual, sobretudo de crianças, instrumentos de avaliação de suporte à aprendizagem em situações de trabalho ainda são escassos, conforme se depreendeu de busca bibliográfica na base de periódicos da Capes entre janeiro e 
junho de 2016. Explorando algumas de suas características psicométricas, principalmente quanto aos fatores medidos e seus itens, as escalas localizadas são analisadas a seguir.

A escala de suportes organizacionais para o desenvolvimento de competências dos funcionários, de Kraimer, Seibert, Wayne, Liden e Bravo (2011), aplicada a 264 funcionários de empresas norteamericanas, compõe-se de seis itens, avaliados em escala de concordância do tipo Likert, a exemplo de: A minha organização oferece oportunidades para os funcionários desenvolverem suas habilidades gerenciais e Minha organização possui programas de desenvolvimento de carreira que ajudam os funcionários a desenvolver seus conhecimentos e habilidades funcionais. Essa escala contribui para o campo por abordar o suporte à aquisição de competências funcionais e gerenciais, mas nenhum de seus itens foca o suporte oferecido por pares, equipes e superiores hierárquicos, tipos de suporte muito evidenciados em estudos anteriores do campo da aprendizagem individual (Coelho et al., 2005; Cheng et al., 2011; Tracey \& Tews, 2005), medindo a percepção de suportes relacionada a aprendizagens formais e informais genéricas de aquisição de competências.

Objetivando medir aspectos contextuais que influenciam a aprendizagem de indivíduos no trabalho, Tracey e Tews (2005) desenvolvem uma escala com 12 itens, classificados em três dimensões, referentes ao apoio recebido de gestores (ex. Os supervisores correspondem às necessidades dos associados para o desenvolvimento pessoal e profissional com oportunidades para participação em treinamentos), da organização (ex. Esta organização oferece excelentes programas de treinamento) e do contexto geral de trabalho (ex. Atribuições de trabalho incluem oportunidades para aprender novas técnicas e procedimentos para melhorar o desempenho). $\mathrm{O}$ instrumento foi validado em amostra de 246 gerentes de restaurantes norte-americanos e, posteriormente, foi revalidado por Choi e Jacobs (2011) em um banco comercial coreano. Embora diferencie suportes oferecidos por gestores e pela organização, a escala em foco dá pouca relevância ao papel desempenhado pelos colegas de trabalho e não diferencia suportes psicossociais de materiais. Além da etapa de aquisição de conhecimentos, o instrumento mede, também, a percepção de suportes à aplicação da competência adquirida, mas trata transferência e aquisição indistintamente, às vezes no mesmo item (ex. Há recompensas e incentivos para aquisição e utilização de novos conhecimentos e habilidades em um posto de trabalho), o que prejudica a avaliação dos resultados.

No contexto brasileiro, não há escalas validadas que tenham como objetivo central os suportes à aquisição de competências. As escalas de suporte à aprendizagem de Coelho (2004) e de Coelho, Abbad e Todeschini (2005), apesar de conterem alguns itens relacionados à aquisição (ex. "Na minha unidade de trabalho há incentivos à busca de novas aprendizagens"; "Meu grupo de trabalho me estimula a buscar novos conhecimentos voltados ao trabalho", Coelho, 2004, p. 106), objetivam, predominantemente, medir os suportes à aplicação das competências em contextos de trabalho.

Ao partir da suposição de que houve aprendizado (resultado), escalas revisadas nesta seção são sensíveis aos enviesamentos provocados pelo tempo, pela memória e por desejabilidade social, além de refletirem o mito de que os trabalhadores aprendem em lugar de trabalhar. Nesse último caso, jogam por terra diferenças cruciais entre (a) situações de trabalho, (b) situações de aprendizagem e (c) aquelas derivadas do grau e qualidade dos suportes à aprendizagem. Tais limitações justificam o desenvolvimento de uma nova medida desse constructo, com foco em experiências específicas de aprendizagem no e para o trabalho.

\section{Suportes organizacionais à transferência}

Os suportes à transferência são apoios gerenciais, sociais, do grupo e organizacionais oferecidos pela organização ao uso de novas habilidades. Algumas ilustrações desses apoios são: oportunidades de práticas das novas habilidades, remoção de obstáculos à transferência de aprendizagem e de acesso às informações, além de apoio material (Figueirêdo, 2008; Hanke, 2006). Itens de suportes psicossociais de escalas de transferência de aprendizagem relacionam-se ao incentivo à aplicação de competências no trabalho, à valorização de tentativas e erros, ao provimento explícito de feedback a tentativas de aplicação do aprendido e à existência de infraestrutura adequada à aplicação dos novos conhecimentos 
no trabalho (Coelho \& Mourão, 2011). A dimensão material dos suportes à transferência, por sua vez, expressa a quantidade, a qualidade e a disponibilidade de recursos materiais e financeiros, e também a qualidade do ambiente físico (Figueirêdo, 2008; Hanke, 2006). Espaço físico, iluminação, mobília, equipamentos são destacados na escala de suporte à aplicação proposta por Brandão, Bahry e Freitas (2008).

A escala de Abbad e Sallorenzo (2001) mensura a aplicação, no trabalho, de aprendizagens em treinamento por meio de 22 itens, distribuídos entre suportes organizacionais, de superiores hierárquicos e de pares. Ressalta-se a ausência de itens que investiguem outros possíveis estímulos à aplicação de competências no trabalho, tais como tolerância a erros e recursos financeiros extras. Embora seja frequentemente apontada por literatura especializada como postura que favorece a aprendizagem (Catino \& Patriota, 2013; March, 1991), tolerância a erros ainda é um item pouco incorporado às escalas revisadas de transferência de aprendizagem. O mesmo não se pode dizer do item recursos financeiros extras. A contribuição da escala de Abbad e Sallorenzo (2001) para o campo é atestada por replicações adaptadas e validadas por Tamayo e Abbad (2006), e por Brandão et al. (2008).

Como já comentado, Coelho et al. (2005) desenvolveram e validaram uma escala de suporte à aprendizagem, tomando como referência um conceito de aprendizagem individual, que se desdobra nas etapas de aquisição, retenção e transferência. Da perspectiva conceitual, essa escala permitiria medir tanto a aquisição quanto a transferência de aprendizagem. Apesar desse esforço conceitual, o instrumento validado é unifatorial, não distinguindo as etapas por fatores, além de limitar-se a medir suportes psicossociais a aprendizagens informais, preterindo medições de aprendizagens formais e suportes materiais. Reaplicada em outros estudos (Balarin et al., 2014; Coelho, Abbad, \& Vasconcelos, 2008), tal escala contribui para o campo ao operacionalizar suportes às aprendizagens naturais e induzidas.

Além de ter itens para medir suportes à aquisição de competências, a escala de Tracey e Tews (2005) também mede a percepção de suportes à aplicação dessas competências no trabalho. São exemplos de itens com essa orientação: Esta organização premia os funcionários que usam novos conhecimentos e habilidades no trabalho; Supervisores reconhecem e estimulam aqueles que aplicam novos conhecimentos e habilidades no trabalho. Os seus itens de suporte não estão agrupados por fase de aquisição e de transferência de aprendizagem, sendo que outros pretendem medir simultaneamente transferência e aquisição (Os funcionários recebem recursos necessários para adquirir e usar novos conhecimentos e habilidades), o que inviabiliza a interpretação dos resultados relativos aos mesmos, conforme já comentado anteriormente.

Instrumento testado em mais de 10 países (Afonso, 2012), inclusive no Brasil (Afonso, 2012; Veloso, Silva, Silva, \& Caetano, 2015), o Learning Transfer System Inventory (LSTI), de Holton, Bates e Ruona (2000), objetiva medir fatores que afetam a transferência de aprendizagem logo após um treinamento específico, constituindo-se de 89 itens e 16 fatores. O LSTI divide-se em dois domínios: (a) relativo ao treinamento específico, com 11 fatores e 63 itens; (b) relativo à organização em geral, com 5 fatores e 26 itens. O primeiro domínio constitui-se em avaliação da percepção do empregado sobre a utilidade do treinamento (ex. Acredito que esta ação me ajudará a desempenhar melhor o meu trabalho). O segundo domínio diz respeito a percepções sobre esforços de transferência, expectativas de desempenho e resultados (ex. O meu desempenho no trabalho melhora quando utilizo o que aprendi), resistência/abertura a mudanças (ex. O meu grupo de trabalho está aberto a mudanças, se isso melhorar o nosso desempenho), e limitações orçamentárias (ex. As limitações orçamentárias no meu trabalho impedirão que eu utilize o que aprendi). Nesse segundo domínio há itens relativos aos aspectos materiais e, principalmente, psicossociais, apesar de não estarem diferenciados. Nos suportes psicossociais não há item para medir o apoio recebido de superiores hierárquicos. O LSTI inclui também itens direcionados a capturar a percepção dos respondentes sobre seu próprio trabalho, sobre equipes e sobre a organização em geral. Do ponto de vista de seu desenho, o LSTI distingue-se positivamente ao viabilizar a coleta de percepções de treinados sobre os fatores que impactam a transferência de aprendizagens formais e específicas dos respondentes. Uma de suas fragilidades é não medir diretamente a transferência, o que limita as possibilidades de estudar as relações entre treinamentos e resultados de 
aprendizagens (Kirwan \& Birchall, 2006). Ademais, ao focar-se em treinamentos formais, o LTSI não permite medir a transferência de aprendizagens informais.

O estudo de Pereira, Loiola e Gondim (2016), desenvolvido em contexto brasileiro, apresentou evidências de validação de escala bifatorial de suporte à transferência composta de oito itens que medem a percepção de professores de um Instituto de Ensino Superior (IES) federal na Bahia sobre suportes materiais e psicossociais à transferência de competências em cinco áreas de atuação (ensino, pesquisa, extensão, gestão e gerais). Nesse caso específico, os pesquisados são instados a avaliar seu posicionamento por meio de uma escala de cinco pontos, em um rol de competências de ensino, de pesquisa, de extensão, de gestão e gerais, dois anos antes e atualmente. Esse procedimento, embora ofereça um foco específico para os pesquisados avaliarem suas aprendizagens e refletirem sobre os suportes recebidos, não especifica a fase de transferência do processo de aprendizagem, induzindo à percepção de que se transfere tudo o que se aprende.

Em suma, a revisão da literatura indica a existência de lacunas nas escalas revisadas de suporte à transferência de aprendizagens para o trabalho, que se limitam a relacionar esses suportes a experiências de treinamento formais, ou que não distinguem os suportes entre as fases de aquisição e de transferência ou, ainda, não medem a transferência em si. Essas lacunas abrem espaço para o desenvolvimento de escalas de suporte que contemplem as múltiplas experiências de transferências de aprendizagens concretas e, simultaneamente, que capturem diferenças entre as etapas de transferência de aprendizagem e de aquisição de aprendizagem.

\section{Estudo 1 - Construção do Inventário de Suporte Organizacional à Aprendizagem (ISOA)}

O Estudo 1 tem por objetivo descrever a construção da ESOAC e da ESOTT e analisar os seus parâmetros de validade e precisão.

\section{Instrumento}

A partir de revisão da literatura de escalas de suporte, procedeu-se à elaboração do Inventário de Suporte Organizacional à Aprendizagem (ISOA), formado pela Escala de Suporte Organizacional à Aquisição de Competências (ESOAC) e pela Escala de Suporte Organizacional à Transferência para o Trabalho (ESOTT). A variável suporte, neste estudo, é reflexiva, tendo sido criada para medir a percepção subjetiva do trabalhador sobre o quanto a empresa oferece suporte. A suposição é que a avaliação subjetiva do trabalhador é fundamental para a efetividade do suporte ofertado. Isto é, não adianta a organização oferecer concretamente suporte material ou gerencial para facilitar seja a aprendizagem de competências, seja a transferência de aprendizagem, se de fato o trabalhador não o percebe desta maneira.

A validação de conteúdo foi realizada por seis juízes, peritos em aprendizagem e competências nas organizações, autores de artigos publicados no Brasil e no exterior e que contribuíram com sugestões referentes ao conteúdo, redação e apresentação dos itens das duas escalas. A partir dessa validação de conteúdo, foram excluídos dois itens redundantes da ESOAC e um item da ESOTT. Para a avaliação semântica, foram realizados dois pré-testes com gestores e funcionários do banco pesquisado, que levaram a ajustes na redação dos enunciados das escalas e de dois itens (um de cada escala), assim como em questões sobre o perfil sócio-demográfico dos respondentes.

Ambas as escalas possuem duas dimensões (suportes materiais e suportes psicossociais), sendo que, em suas versões iniciais, a ESOAC foi composta por nove itens, e a ESOTT por 14 itens, medidos em uma escala de 1 a 5 pontos. Inicia-se a aplicação da ESOAC e da ESOTT estimulando o respondente a avaliar experiências próprias de aprendizagem e de transferência de 15 competências gerenciais, funcionais e organizacionais, extraídas de documentos da organização pesquisada e que estão listadas nas perguntas de abertura do inventário. $\mathrm{O}$ ato de avaliar o que foi aprendido, em determinado espaço 
de tempo, e em que medida o aprendizado foi aplicado, tem por objetivo direcionar a avaliação dos suportes recebidos a experiências específicas, não genéricas, minimizando lacuna detectada por Kirwan e Birchall (2006) e contribuindo para a redução de vieses de tempo, de memória e de desejabilidade social recorrentes no campo.

\section{Participantes e procedimentos de coleta de dados}

A Universidade Corporativa da instituição enviou o questionário pela intranet a 1.213 funcionários, selecionados por critérios de conclusão em cursos de competências gerenciais e processos de negócios promovidos pela organização. Apesar desse recorte dos respondentes, o instrumento não se limita a coletar informações sobre aprendizagens formais. Desse total, 292 funcionários aceitaram e deram início ao processo de responder o questionário, indicando uma taxa de retorno de cerca de $24 \%$, satisfatória para esse tipo de pesquisa. Após avaliação de qualidade, 203 questionários compuseram a amostra final.

Do total de participantes (203), 141 são homens e 62 mulheres. De perfil jovem, 72,4\% dos respondentes têm até 40 anos de idade. A maioria deles $(75,9 \%)$ apresenta entre dois e dez anos de trabalho na instituição pesquisada. Em relação à escolaridade, 48,7\% têm o nível superior completo, $38,9 \%$ são especialistas, $5,4 \%$ mestres e $1 \%$ doutor. Quanto ao cargo, a maioria dos respondentes é analista bancário (88,2\%), enquanto $2 \%$ são analistas técnicos e $20 \%$ especialistas. Por fim, quanto à função desempenhada, 67\% são gerentes (principais, de agência/unidade, ou intermediária, executiva/de negócios), 10,8\% coordenadores ou assessores e 22,1\% ocupam outras funções técnicas.

\section{Procedimentos de análise de dados}

As percepções de aquisição e de transferência de competências foram medidas por teste $t$ de médias e por desvio-padrão. Competências adquiridas pelos indivíduos foram definidas como aquelas cujas diferenças de médias entre domínio anterior e domínio atual foram estatisticamente significativas $(p<0,05)$. Já a transferência de competências foi considerada existente quando a média de aplicação de competências foi maior ou igual à média de domínio atual da competência aprendida.

Análises fatoriais exploratórias (AFEs) e de consistência interna foram realizadas em ambas as medidas. Nas AFEs, o método de extração foi o da Análise dos Eixos Principais (Principal Axis Factoring) com rotação oblíqua entre os fatores (OBLIMIN), uma vez que é expectável que as dimensões a emergir estejam correlacionadas em ambas as escalas. Alfa de Cronbach maior que 0,6 foi o parâmetro de confiabilidade adotado (Hair, Black, Babin, Anderson, \& Tatham, 2009).

\section{Resultados}

O procedimento prévio adotado na ESOAC e na ESOTT, que direciona o respondente a indicar seu nível de domínio em relação a um rol de 15 competências específicas do seu ambiente de trabalho, indicou que os funcionários do banco pesquisado aprenderam e aplicaram competências nos últimos dois anos.

Quanto ao nível de aprendizagem, os resultados (média do domínio da competência há dois anos; média de domínio atual; diferença entre as médias; desvio-padrão da diferença entre as médias; nível de significância da diferença) indicaram que houve aprendizagem significativa de competências organizacionais $(3,75 ; 4,33 ; 0,58$; sd 0,$61 ; p<0,001)$, de competências gerenciais $(3,05 ; 3,93 ; 0,87$; sd $0,62 ; p<0,001)$ e de competências funcionais $(3,16 ; 3,92 ; 0,76$; sd 0,$53 ; p<0,001)$. Para o teste de todos esses pares, considerou-se um grau de liberdade $(\mathrm{df})=202$. Quanto ao nível de aplicação no trabalho das competências aprendidas, os resultados indicaram que os respondentes aplicam competências organizacionais $(4,33 ; 4,33 ; 0,00 ;$ sd 0,$43 ; p=0,957)$, competências gerenciais $(3,93$; $3,93 ; 0,00 ;$ sd 0,$63 ; p=0,910)$ e competências funcionais $(3,92 ; 3,98 ; 0,06 ;$ sd 0,$44 ; p=0,053)$. 


\section{Escala de suporte organizacional à aquisição de competências (ESOAC)}

Os resultados da aplicação da AFE indicaram que os itens da ESOAC Incentivos relacionados à ascensão profissional na empresa e Recursos financeiros extras ou outros incentivos financeiros foram eliminados porque apresentaram, respectivamente, comunalidades e cargas fatoriais abaixo dos valores aceitáveis. Em seguida, aplicou-se outra AFE aos itens remanescentes (Tabela 1). Convergindo com a teoria, os resultados revelam a existência de dois fatores (material e psicossocial), que explicam $71,01 \%$ da variância dos itens. O fator suporte material apresentou alfa de Cronbach de 0,842 , enquanto o fator suporte psicossocial assumiu valor de 0,677 , sendo que ambos os valores denotam confiabilidade satisfatória.

Tabela 1

Cargas Fatoriais da Escala de Suporte Organizacional à Aquisição de Competências (ESOAC)

\begin{tabular}{|c|c|c|c|c|}
\hline Fatores & Itens & Fator 1 & Fator 2 & Comunalidades \\
\hline \multirow[t]{3}{*}{$\begin{array}{l}\text { Suporte Material à } \\
\text { Aprendizagem }\end{array}$} & $\begin{array}{l}\text { Recursos materiais (estrutura física e } \\
\text { tecnológica) em QUANTIDADE suficiente. }\end{array}$ & ,933 & & ,814 \\
\hline & $\begin{array}{l}\text { Recursos materiais (estrutura física e } \\
\text { tecnológica) com QUALIDADE suficiente. }\end{array}$ & ,945 & & ,841 \\
\hline & $\begin{array}{l}\text { Cursos, treinamentos, oficinas de trabalho ou } \\
\text { similares. }\end{array}$ &, 529 & & ,464 \\
\hline \multirow[t]{7}{*}{$\begin{array}{l}\text { Suporte Psicossocial } \\
\text { à Aprendizagem }\end{array}$} & $\begin{array}{l}\text { Palavras de estímulos de colegas de trabalho } \\
\text { para me engajar na aprendizagem de novas } \\
\text { ideias/formas de executar atividades. }\end{array}$ & & ,777 &, 555 \\
\hline & $\begin{array}{l}\text { Palavras de estímulo de superiores } \\
\text { hierárquicos para me engajar na aprendizagem } \\
\text { de novas ideias/formas de executar atividades. }\end{array}$ & &, 773 & ,628 \\
\hline & $\begin{array}{l}\text { Flexibilidade na jornada de trabalho para } \\
\text { buscar novas aprendizagens. }\end{array}$ & & 323 & ,264 \\
\hline & Correlação interfatores & ,842 & ,417 & \\
\hline & Alfa de Cronbach & & 677 & \\
\hline & Número de itens & 03 & 03 & \\
\hline & Variância Explicada & $49,99 \%$ & $21,04 \%$ & \\
\hline
\end{tabular}

As cargas fatoriais de cinco itens que compõem a ESOAC apresentaram valores acima de 0,5. Já o item flexibilidade na jornada de trabalho para buscar novas aprendizagens é o único que apresentou carga fatorial abaixo do padrão elevado obtido pelos outros itens. Finalmente, o fator material e o psicossocial são correlacionados, indicando que quanto maior é o suporte material à aquisição de competências, maior também é o suporte psicossocial a esta etapa da aprendizagem (Tabela1), corroborando-se, mais uma vez, resultados de trabalhos anteriores.

\section{Escala de suporte organizacional à transferência para o trabalho (ESOTT)}

Os procedimentos de validação da ESOTT foram similares aos adotados para a escala anterior, iniciando-se com a aplicação de AFEs. Os resultados indicaram a necessidade de excluir os seguintes itens que apresentaram cargas fatoriais muito baixas e/ou comunalidades: Mais oportunidades de ascensão profissional, Palavras de estímulo de superiores hierárquicos e Auxilio de colegas de trabalho e elogios de colegas de trabalho. Após exclusões dos itens, reaplicou-se a AFE (Tabela 2). Os resultados também indicam a existência de dois fatores, material e psicossocial, que explicam $66 \%$ da variância dos itens. Quanto à confiabilidade, os valores do alfa de Cronbach apresentados em ambos os fatores 
são satisfatórios: o fator material apresentou alfa de Cronbach de 0,811 , enquanto o fator psicossocial assumiu o valor de 0,835 (Tabela 2 ).

Tabela 2

Cargas Fatoriais da Escala de Suporte Organizacional à Transferência para o Trabalho (ESOTT)

\begin{tabular}{|c|c|c|c|c|}
\hline Fatores & Itens & Fator 1 & Fator 2 & Comunalidades \\
\hline \multirow[t]{4}{*}{$\begin{array}{l}\text { Suporte Material } \\
\text { à Transferência }\end{array}$} & $\begin{array}{l}\text { Recursos materiais (estrutura física e } \\
\text { tecnológica) em QUANTIDADE suficiente. }\end{array}$ & 895 & & ,747 \\
\hline & $\begin{array}{l}\text { Recursos materiais (estrutura física e } \\
\text { tecnológica) com QUALIDADE suficiente. }\end{array}$ & ,913 & & ,770 \\
\hline & $\begin{array}{l}\text { Recursos financeiros extras ou outros } \\
\text { incentivos financeiros. }\end{array}$ &, 508 & & ,376 \\
\hline & Oportunidades de aplicação prática & ,467 & & ,383 \\
\hline \multirow{7}{*}{$\begin{array}{l}\text { Suporte } \\
\text { Psicossocial à } \\
\text { Transferência }\end{array}$} & $\begin{array}{l}\text { Auxílio de superiores hierárquicos para } \\
\text { planejar e remover obstáculos. }\end{array}$ & &, 613 &, 615 \\
\hline & Elogios de superiores hierárquicos. & &, 705 & ,637 \\
\hline & Palavras de estímulo de colegas de trabalho. & & ,957 & 802 \\
\hline & $\begin{array}{l}\text { Tolerância aos erros que cometo ao por em } \\
\text { prática competências aprendidas }\end{array}$ & &, 521 &, 351 \\
\hline & $\begin{array}{l}\text { Correlação } \\
\text { Alfa de Cronbach }\end{array}$ &, 811 & $\begin{array}{l}, 410 \\
, 835\end{array}$ & \\
\hline & Número de itens & 04 & 04 & \\
\hline & Variância Explicada & $49,10 \%$ & $16,90 \%$ & \\
\hline
\end{tabular}

As cargas fatoriais dos itens do fator material apresentaram valores satisfatórios com comunalidades elevadas. O mesmo se verifica no fator psicossocial. Estes resultados evidenciam a adequação dos itens da ESOTT, indicando sua validade fatorial. Assim como na escala anterior, o coeficiente de correlação entre eles foi de 0,410 , indicando que a percepção do suporte material está relacionada à percepção do suporte psicossocial, ou seja, quanto mais o indivíduo percebe que a organização provê suporte material à transferência, mais forte também é sua percepção de suporte psicossocial.

\section{Discussão}

De modo geral, a ESOAC e a ESOTT apresentaram indicadores satisfatórios de validade e fiabilidade, confirmando-se sua estrutura bifatorial. A composição de itens das duas escalas apresenta relativa diferenciação, tanto no fator psicossocial quanto no material. Na ESOAC, além dos itens relativos à qualidade e à quantidade de recursos materiais disponibilizados, presentes também na ESOTT, a oferta de: Cursos, treinamentos, oficinas de trabalho e similares é um item que diferencia o seu fator material do mesmo fator da ESOTT. No caso dos itens dos fatores psicossociais de ambas as escalas, as diferenças são mais marcantes. Em comum, ambas as escalas só possuem o item Palavra de estímulo de colegas de trabalho. Os outros itens do fator psicossocial da ESOTT - Auxílio de superiores hierárquicos para planejar e remover obstáculos, Elogios de superiores e Tolerância a erros são exclusivos dessa escala. Chama-se a atenção para o fato de que os itens do fator psicossocial da ESOAC são mais dirigidos a incentivar o engajamento em situações de aprendizagem, ao passo que os itens do fator psicossocial da ESOTT se dividem entre itens que estimulam os aprendizes a aplicar o que 
aprenderam e itens que procuram dar sustentação e segurança psicológica aos trabalhadores para aplicarem o que aprenderam.

Ainda quanto aos itens validados, ressalta-se a decisão de não excluir o item Flexibilidade na jornada de trabalho para buscar novas aprendizagens do fator psicossocial da ESOAC, mesmo tendo apresentado carga fatorial menor que os demais itens do fator. Tal decisão deveu-se a importância do manejo do tempo (Cheng et al., 2011; Coelho et al., 2005; Lohman, 2009), além da possibilidade de vir a testar novamente a estrutura dos itens do fator em um segundo estudo. Já o item Recursos financeiros extras ou outros incentivos financeiros não obteve carga fatorial suficiente para ser incluído, diferente do que ocorreu em estudos anteriores que o incorporam na composição dos suportes materiais (Choi \& Jacobs, 2011; Ellinger, 2005; Lee et al., 2004; Tracey \& Tews, 2005). A exclusão desse item vai ao encontro, entretanto, dos resultados da pesquisa de Lohman (2009) que indicam que a ausência de incentivos financeiros não é fator inibidor importante para o envolvimento em atividades informais de aprendizagem. Essa última proposição parece consistente com o fato de que aprendizagens informais se relacionam mais à exploitation de conhecimentos e de recursos já existentes na organização (Argyris \& Schon, 1974; March, 1991) e tendem a ser motivadas para superar dificuldades vivenciadas ao longo da jornada de trabalho.

Na ESOTT, o item Recursos financeiros extras ou outros incentivos financeiros apareceu no fator material de suporte à transferência de competências para o trabalho, o que replica resultados de estudos anteriores (Hanke, 2006; Kraimer et al., 2011; Tracey \& Tews, 2005). O item Oportunidades de aplicação prática também foi mantido após apresentar valor abaixo dos demais, com respaldo da teoria e também porque a mesma escala será retestada ainda neste trabalho.

Apesar de encontrar suporte na teoria, a manutenção de itens na ESOAC e na ESOTT que apresentaram valores um pouco abaixo dos parâmetros de análises fixados estimula a realização de um segundo estudo com o objetivo de replicar o primeiro para testar a adequação da estrutura fatorial proposta.

\section{Estudo 2 - Análises Confirmatórias da ESOAC e da ESOTT}

O Estudo 2 tem por objetivo replicar os resultados do Estudo 1 numa segunda amostra de trabalhadores em uma Organização Não Governamental (ONG) que atua no Nordeste do Brasil. Para tanto, este estudo aplica Análises Fatoriais Confirmatórias para que seja verificada a adequação do modelo de medida proposto para ambas as escalas.

\section{Instrumento}

A versão final do Inventário de Suporte Organizacional à Aprendizagem (ISOA), após análise exploratória do estudo 1, compõe-se por seis itens da ESOAC e oito da ESOTT, e não recebe qualquer alteração no teste do Estudo 2. Assim como no estudo 1, os participantes foram estimulados a avaliar seus níveis de aprendizagem e de transferência de aprendizagem de competências gerenciais e organizacionais extraídas de documentos da ONG. A pesquisa foi realizada em junho de 2015.

\section{Participantes e procedimentos de coleta}

A ONG promoveu o envio do questionário, via intranet, a 1.300 empregados. Desse total, 295 responderam, o que representou $22,7 \%$ da população total. Depois de excluir 43 unidades preenchidas de modo incompleto, participaram 252 empregados da $\mathrm{ONG}$, o que representou quase $20 \%$ da população total. Esse percentual é considerado satisfatório neste tipo de pesquisa. Os participantes distribuíram-se entre coordenadores $(65,1 \%)$, assessores técnicos $(18,3 \%)$, assistentes $(14,7 \%)$, analistas $(1,2 \%)$, gerentes $(0,4 \%)$ e diretores $(0,4 \%)$. Desses, $52,4 \%$ são do sexo feminino e $47,6 \%$ masculino. Apenas 
$7,9 \%$ dos respondentes têm mais de 40 anos de idade, os demais se enquadram nas faixas etárias entre 20 e $30(48 \%)$ e entre 31 e 40 (44,1\%). Observa-se, portanto, o mesmo perfil jovem da amostra do estudo anterior. A maioria tem ensino superior completo (69,4\%), enquanto 12,7\% têm título de especialização. Nenhum participante possui mestrado ou doutorado. Quanto ao tempo de trabalho na organização, a maioria $(69,1 \%)$ tem entre 3 e 10 anos.

\section{Procedimentos de análise de dados}

Assim como no primeiro estudo, foram medidas com base em teste $t$ de médias e desvio-padrão as percepções de aquisição e de transferência de competências. Na ONG, 12 competências foram selecionadas em um rol de competências de gestão, negocial, operacional e técnicas.

As análises fatoriais confirmatórias (AFC) foram realizadas por meio do AMOS 18.0. A base de dados não contém casos omissos nem outliers extremos univariados ou multivariados, e os resíduos dos indicadores são normalmente distribuídos. Por esta razão, os parâmetros foram estimados com base na matriz de variâncias-covariâncias dos indicadores, adotando-se o método da Máxima Verossimilhança. A adequação dos modelos fatoriais propostos foi aferida pelos seguintes índices de ajustamento (Byrne, 2010): a razão do qui-quadrado pelos graus de liberdade $\left(\chi^{2} / \mathrm{gl}\right)$, assumindo-se coeficientes menores do que 5 como indicadores de ajuste adequado; Goodness-of-Fit Index (GFI), Adjusted Goodness-of-Fit Index (AGFI) e Comparative-FIT-Index (CFI), assumindo-se coeficientes superiores a 0,90 como indicadores de adequação do modelo; e Root Mean Square Error of Approximation (RMSEA), em que valores de 0,08 ou menores indicam ajuste satisfatório dos modelos aos dados.

\section{Resultados}

Os resultados do procedimento adotado antes da aplicação da ESOAC e da ESOTT indicaram que houve aprendizagem e aplicacação do conjunto de doze competências (três gerenciais e nove organizacionais) analisadas na ONG, uma vez que o teste $t$ de médias apresentou percepção significativa de aprendizagem em todas as competências por parte dos respondentes. Aqui, destacam-se os resultados por grupo de competências: organizacionais (média domínio antes: 3,54; média domínio depois: 4,21; diferença entre as médias: 0,67 ; sd 0,$62 ; p<0,001)$ e gerenciais $(3,27 ; 4,03 ; 0,75 ; \mathrm{sd} 0,73 ; p<0,001)$. Considera-se, nesses testes, grau de liberdade $(\mathrm{df})=252$ para cada uma das competências.

O Estudo 2 também concluiu que o nível de aplicação das competências no trabalho não é significativamente inferior ao nivel de aprendizagem, ou seja, os funcionários da ONG percebem que aplicam no trabalho as competências organizacionais $(4,21 ; 4,33 ; 0,11$; sd 0,$27 ; p<0,001)$ e as competências gerenciais $(4,03 ; 4,13 ; 0,10 ; \mathrm{sd} 0,35 ; p<0,001)$ aprendidas.

\section{Análise fatorial confirmatória da ESOAC}

A AFC aplicada à ESAOC testa uma estrutura fatorial composta por dois fatores correlacionados (Suporte Material e Suporte Psicossocial), sendo cada fator medido por três itens. Os resultados mostram bons indicadores de ajustamento do modelo proposto aos dados: $\chi^{2}(13)=53,37 ; \mathrm{p}<0,001 ; \chi^{2} / \mathrm{gl}=6,67$; $\mathrm{GFI}=0,94 ; \mathrm{AGFI}=0,85 ; \mathrm{CFI}=0,94 ; \mathrm{RMSEA}=0,150(\mathrm{IC} 90 \%=0,114 ; 0,190)$. Igualmente importante é o fato de as cargas fatoriais se mostrarem fortes. De fato, os itens têm cargas fatoriais variando de 0,52 a 0,95 , o que indica a adequação de cada item para medir o seu respectivo fator. A variância extraída média (VEM) revelou-se também adequada, sendo de 0,66 para o Suporte Material, e 0,43 para o Suporte Psicossocial. Também importante é o fato de a média das VEMs (i.e., .55) ser maior do que o quadrado da correlação interfatores $\left(r^{2}=.48\right)$, o que advoga a favor da validade discriminante entre os fatores. Os dois fatores mostram-se fortemente correlacionados, indicando que quanto maior é o suporte material, também maior é o suporte psicossocial (Figura 1). 


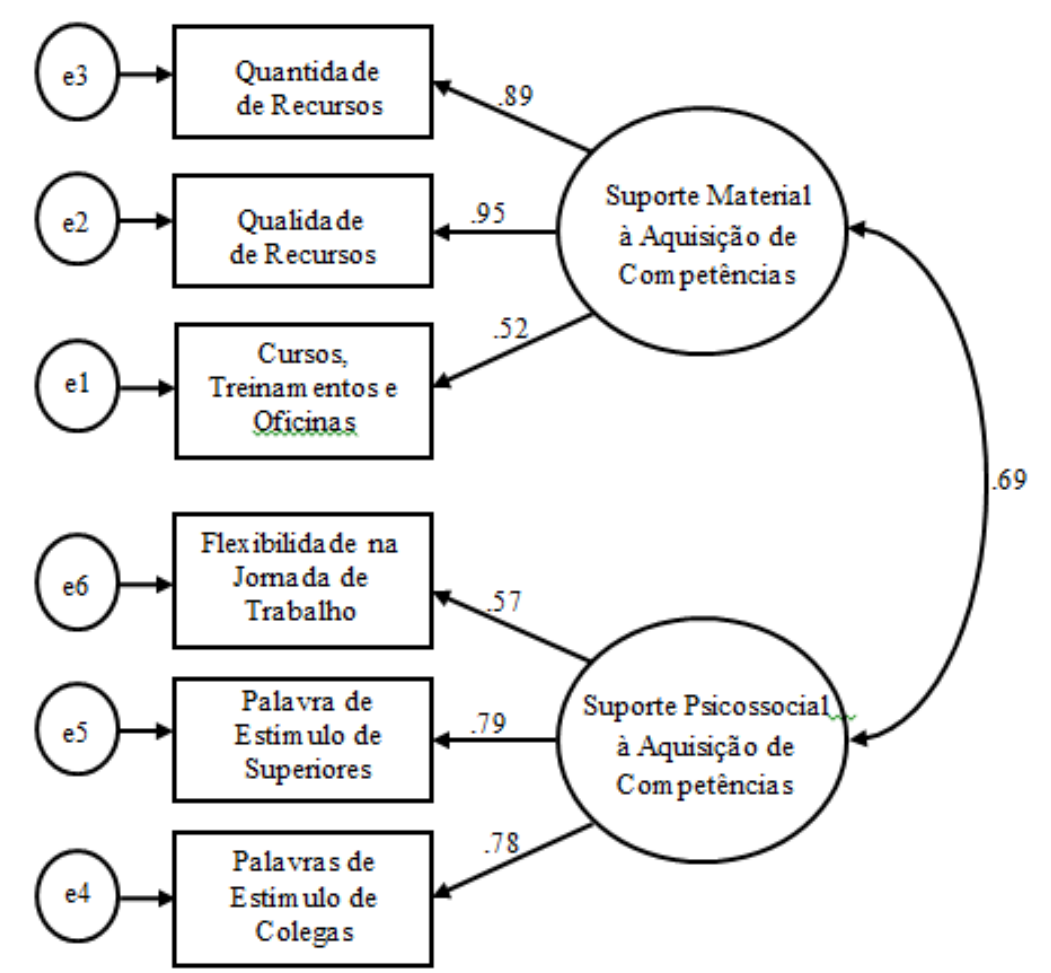

Figura 1. Estrutura Fatorial Confirmatória da ESOAC

\section{Análise fatorial confirmatória da ESOTT}

A AFC aplicada à ESOTT também testa uma estrutura fatorial composta por dois fatores correlacionados (Suporte Material à Transferência e Suporte Psicossocial à Transferência), sendo cada fator medido por quatro itens. Os resultados mostram indicadores de ajustamento do modelo aos dados satisfatórios: $\chi^{2}(19)=112,35 ; \mathrm{p}<0,001 ; \chi^{2} / \mathrm{gl}=5,91$; GFI $=0,89$; AGFI $=0,80 ;$ CFI $=0,93$; RMSEA $=0,140(\mathrm{IC} 90 \%=0,120 ; 0,170)$. A análise suplementar aos índices de ajustamento sugeriu a presença de correlação entre os resíduos de dois itens do fator Suporte Material à Transferência, conforme especificado na Figura 2. 


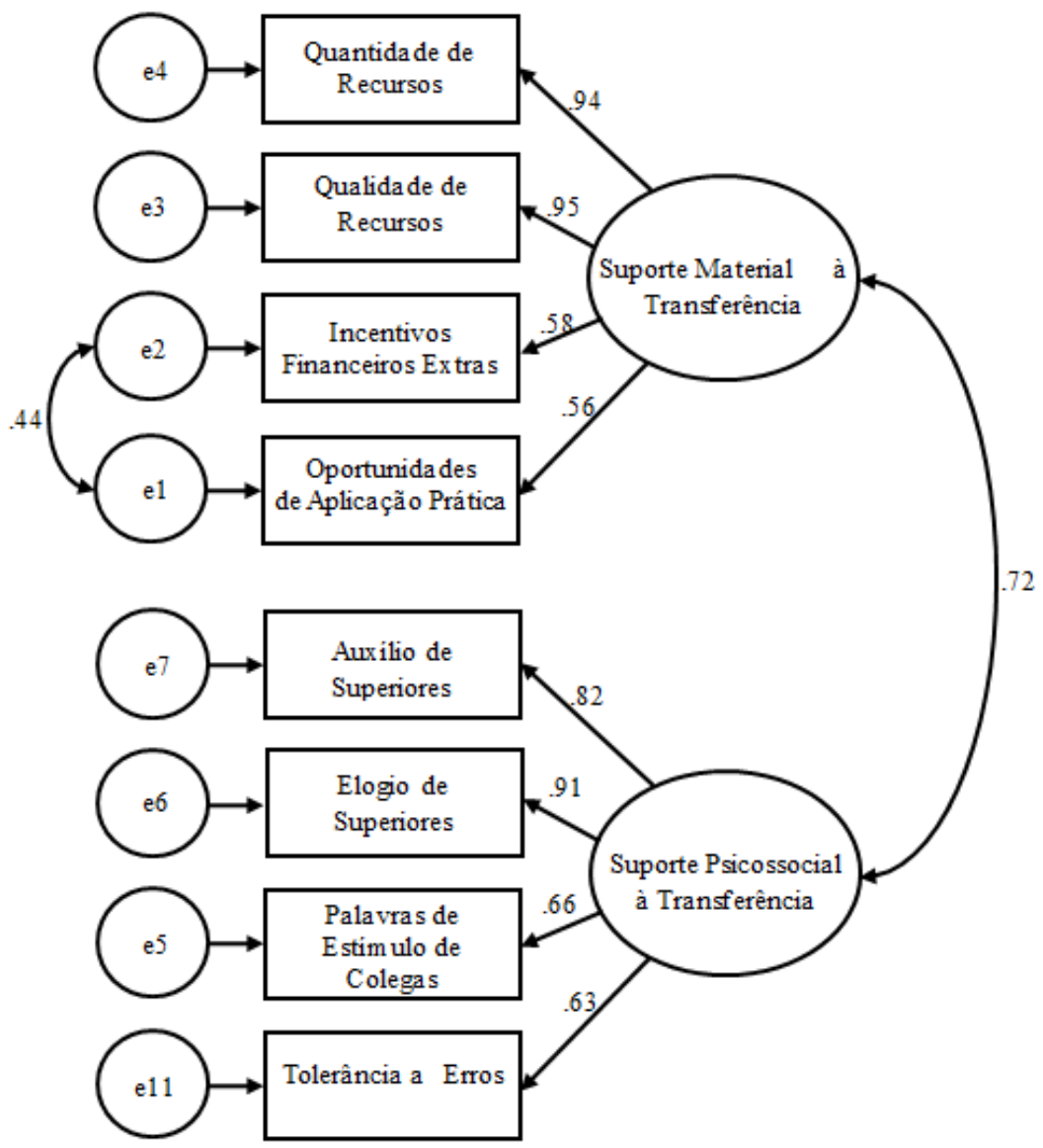

Figura 2. Estrutura Fatorial Confirmatória da ESOTT

Após a implementação da correlação entre os dois resíduos indicados, o modelo passou a ter ajustamento muito bom aos dados: $\chi^{2}(18)=60,80 ; \mathrm{p}<0,001 ; \chi^{2} / \mathrm{gl}=3,38$; GFI = 0,95; AGFI = 0,89; $\mathrm{CFI}=0,97 ;$ RMSEA $=0,097$ (IC90\% $=0,071 ; 0,125)$. A análise das cargas fatoriais indica a presença de coeficientes muito fortes, variando de 0,52 a 0,95 no fator suporte material, e de 0,57 a 0,79 no fator suporte psicossocial. Os fatores VEM material e psicossocial estão fortemente correlacionados, de modo que quanto maior é a percepção de suporte material, maior também é a percepção de suporte psicossocial (Figura 2). A análise das VEMs revelou ser igual a 0,61 para o Suporte Material à Transferência, e 0,58 para o Suporte Psicossocial à Transferência. O importante é o fato de a média das VEMs (i.e., .60) ser maior do que o quadrado da correlação interfatores $\left(r^{2}=.52\right)$, indicando que, embora fortemente correlacionados, há evidência de validade discriminante entre os fatores.

No conjunto, os resultados apresentados confirmam a estrutura fatorial das duas escalas, verificada no Estudo 1, e a adequação das medidas aqui propostas para medir o suporte à aprendizagem de competências e à transferência dessa aprendizagem.

\section{Discussão}

A análise fatorial confirmatória apresentou resultados satisfatórios no que diz respeito à validade e à fiabilidade das medidas inicialmente propostas no Estudo 1, ratificando sua estrutura bifatorial. Os itens que diferenciam as duas medidas foram confirmados nas duas escalas, replicando-se aqui as descrições e argumentações que mostram diferenças de conteúdo das duas escalas e, sobretudo, a importância de itens que refletem práticas com fins de despertar percepções de segurança psicológica em situações de aplicação no trabalho de competências aprendidas, a exemplo de Tolerância a erros e auxílio de superiores no planejamento e remoção de obstáculos à aplicação.

$\mathrm{Na}$ ESOAC, o item Flexibilidade na jornada de trabalho para buscar novas aprendizagens, que apresentou carga fatorial abaixo do conjunto dos outros itens em seu fator, apresentou, no estudo 2, 
carga fatorial de 0,57 , indicando a sua adequação para medir o suporte psicossocial à aprendizagem. A presença desse item confirma os estudos de Lohman (2009), Coelho et al. (2005) e Cheng, Wang, Yang e Peng (2011), que mencionam a variável tempo como relevante para o suporte à aquisição de novas competências. Também convergiu com a literatura a validação de itens relacionados a palavras de estímulo de superiores hierárquicos (Balarin et al., 2014; Clarke, 2004; Coelho et al., 2005; Ellinger, 2005) e de colegas de trabalho (Balarin et al., 2014; Cheng et al., 2011; Coelho et al., 2005; Lohman, 2005). O fator material da ESOAC é composto por itens que reforçam aspectos de estudos anteriores, tal como a promoção de "cursos, treinamentos e oficinas" para indivíduos adquirirem competências. As escalas de suportes propostas por Tracey e Tews (2005) e por Kraimer et al. (2011) já haviam destacado a importância desse item.

Na dimensão material da ESOTT, o item Oportunidades para aplicação prática, que no Estudo 1 havia apresentado baixa carga fatorial, apresentou carga fatorial satisfatória de 0,56. A relevância desse item já havia sido destacada nas escalas de suportes de Tracey e Tews (2005), e de Coelho et al. (2005). A dimensão psicossocial da ESOTT é composta por itens relacionados a apoios de superiores hierárquicos e colegas de trabalho, assim como nos estudos de Tracey e Tews (2005), Coelho et al. (2005), Brandão et al. (2008), Choi e Jacobs (2011) e Balarin, Zerbini e Martins (2014). Ademais, o fator psicossocial da ESOTT contém o item Tolerância a erros, já utilizado na escala proposta por Coelho et al. (2005), porém ainda pouco considerado em outras escalas de suporte à aprendizagem. Brandão et al. (2008) tratam erros em sua escala de suporte, entretanto, no sentido de restrição aos erros e não de tolerância. Em seu estudo de natureza qualitativa, Catino e Patriota (2013) valorizam a relevância da tolerância aos erros para aplicação de competências no trabalho.

Em geral, o desenho da ESOAC e da ESOTT e, sobretudo, a decisão de colocar uma pergunta que as antecede sobre as competências aprendidas e transferidas pelos respondentes permitem elidir fragilidades identificadas em revisão de bibliografia, tais como: medir suporte sem se medir aquisição e aplicação de competências individuais efetivamente, reforçar vieses de tempo e de desejabilidade social, impedir a diferenciação entre os tipos de suportes a processos de aprendizagem no e para o trabalho.

\section{Conclusões}

Amparando-se em conceitos cognitivistas de aprendizagem e de transferência de aprendizagem de competências e em resultados de revisões de escalas de suportes, a presente pesquisa ${ }^{(1)}$ apresentou duas novas escalas de medida dos suportes disponibilizados pela organização para que os indivíduos aprendam competências, adquirindo conhecimentos, habilidades e atitudes, e as transfiram para o trabalho nas organizações. A aprendizagem de competências foi definida como a aquisição de CHA's (conhecimentos, habilidades e atitudes) pelos indivíduos por meio de processos formais (comparativamente mais estruturados e mais direcionados pela organização) e informais (comparativamente menos estruturados e mais sob o controle dos indivíduos). Quando as competências aprendidas são aplicadas no trabalho, verifica-se a transferência de aprendizagem, que gera possíveis mudanças de comportamentos no trabalho, impactando o desempenho de trabalhadores, equipes de trabalho e, consequentemente, da organização.

Suportes à aprendizagem compreendem um conjunto de práticas de apoio aos processos de aprendizagem dos indivíduos. De igual modo, suportes à transferência correspondem a um conjunto de práticas de apoio à transferência de aprendizagem, ou melhor, à aplicação no trabalho das competências adquiridas pelos indivíduos.

Os resultados dos testes das duas escalas apresentam evidências de validade da estrutura fatorial das medidas compostas por dois fatores: psicossocial e material. A natureza bifatorial de ambas as escalas corrobora a estrutura desses tipos de apoios já evidenciada em outros estudos. Sustentou-se, com base na literatura, que ambos os suportes se referem a fenômenos diferentes. A composição dos itens de 
cada fator das escalas com evidências de validação reforça essa postulação de diferenças entre os dois tipos de fatores.

Este estudo contribui para o trabalho de praticantes e pesquisadores. Na medida em que estimula os entrevistados a evocarem experiências específicas e interrelacionadas de aprendizagem e de transferência de competências, mitigam-se riscos de vieses de tempo, de memória e de tendência a dar respostas socialmente desejadas. Também tal estratégia de operacionalização permite relacionar os suportes aos resultados da aprendizagem e das transferências (o que foi aprendido e o que foi transferido). Aplica-se ainda à investigação de suportes seja em experiências concretas de aprendizagem formal, seja informal. Adicionalmente, ao medir aquisição e transferência de competências, viabiliza especificar os suportes por fase do processo de aprendizagem (aquisição e transferência), independentemente do tipo de aprendizagem, se formal ou informal. Contribui também para superar a limitação do tamanho das amostras das pesquisas, realizando dois estudos, um exploratório e outro confirmatório, em organizações distintas. As duas amostras foram suficientes para recolher evidências de validação das escalas, embora se reconheça que um maior número de observações poderia reforçar ainda mais a evidência de validade e precisão do inventário. Todas essas características do instrumento contribuem para aumentar sua acurácia na medição dos fenômenos, o que é positivo tanto para praticantes quanto para estudiosos.

Admite-se como limitação da pesquisa que o método de recolha de dados usado introduz um viés de cobertura, pois capta apenas as percepções das pessoas motivadas a responderem o questionário por meio eletrônico, não sendo capaz de acessar aquelas que prefeririam respondê-lo em papel e lápis, nem as que prefeririam fazê-lo por meio de entrevistas presenciais ou por telefone. Apenas investigações usando multi-mode methods reduziria esse tipo de viés, porém seriam muito mais dispendiosas para, eventualmente, mostrarem o mesmo padrão de resultados aqui obtidos.

A opção por medidas reflexivas de suporte representa outra limitação do presente estudo. Estudos futuros podem explorar a combinação entre medidas reflexivas e observações ou, ainda, substituir as medidas reflexivas por formativas que poderiam ser criadas com base na quantidade e na qualidade dos suportes dados pelas empresas. No entanto, não se pode negligenciar a defesa anteriormente feita da medida reflexiva porque as organizações podem oferecer suportes à aprendizagem e à transferência de aprendizagem, mas os funcionários podem não percebê-los como tais.

Outra fonte de limitação pode ser a utilização do mesmo procedimento de resposta para os participantes (i.e., respostas a escalas de Rating). No entanto, no caso deste estudo, o enviesamento produzido pelo Common Method Variance (CMV) não pode explicar os resultados, porque se isto de fato ocorresse, os resultados da análise fatorial exploratória no estudo 1 teriam mostrado a presença de um único fator em cada medida, o que não foi o caso. Dois fatores foram claramente identificados com justificativa teórica. Adicionalmente, os resultados das VEMs, calculados com base nos parâmetros estimados na análise fatorial confirmatória, reforçam a evidência de que o CMV não representa uma ameaça à interpretação conceptual defendida. Se assim o fosse, os valores das VEMs teriam indicado a presença de um único fator, exatamente o CMV.

Sugere-se, para estudos futuros, a aplicação da ESOAC e da ESOTT no intuito de investigar possíveis efeitos de predições, moderações ou mediações para com diferentes constructos relacionados ao campo da aprendizagem em contexto organizacional, além de recolher novas evidências empíricas sobre as diferenças entre as duas escalas.

\section{Nota} ${ }^{1}$ Pesquisa apoiada pela Coordenação de Aperfeiçoamento de Pessoal de Nível Superior (CAPES) por meio de bolsa de estudos
ao primeiro autor, Bruno Chaves Correia-Lima. 


\section{Referências}

Abbad, G., \& Sallorenzo, L. H. (2001). Desenvolvimento e validação de escalas de suporte à transferência de treinamento. Revista de Administração da Universidade de São Paulo, 36(2), 3345.

Afonso, C. C. (2012). Validação fatorial e preditiva no Brasil do instrumento de Avaliação do Sistema de Transferência de Aprendizagem (ASTA) (Dissertação de mestrado). Universidade de Brasília, Brasília, DF, Brasil.

Argyris, C., \& Schon, C. D. A. (1974). Theory in practice. San Francisco: Jossey-Bass.

Balarin, C. S., Zerbini, T., \& Martins, L. B. (2014). A relação entre suporte à aprendizagem e impacto de treinamento no trabalho. Revista Eletrônica de Administração, 20(2), 341-370. Recuperado de http://www.scielo.br/pdf/read/v20n2/1413-2311-read-20-02-0341.pdf. http://dx.doi.org/10.1590/1413-2311017201341925

Baranik, L. E., Roling, E. A., \& Eby, L. T. (2010). Why does mentoring work? The role of perceived organizational support. Journal of Vocational Behavior, 76(3), 366-373. http://dx.doi.org/10.1016/j.jvb.2009.07.004

Bauer, T. N., \& Green, S. G. (1998). Testing the combined effects of newcomer information seeking and manager behavior on socialization. Journal of Applied Psychology, 83(1), 72. http://dx.doi.org/10.1037/0021-9010.83.1.72

Blume, B. D., Ford, J. K., Baldwin, T. T., \& Huang J. L. (2010). Transfer of training: A meta-analytic review. Journal of Management, 36(4), 1065-1105. http://dx.doi.org/10.1177/0149206309352880

Brandão, H. P., Bahry, C. P., \& Freitas, I. A. (2008). Os impactos do suporte à transferência sobre a aplicação de competências no trabalho: A percepção dos mestres e doutores do Banco do Brasil. Revista de Administração, 43(3), 224-237.

Brandão, H. P., Borges-Andrade, J. E., \& Guimarães, T. de A. (2012). Desempenho organizacional e suas relações com competências gerenciais, suporte organizacional e treinamento. Revista de Administração, 47(4), 523-539. http://dx.doi.org/10.5700/rausp1056

Byrne, B. M. (2013). Structural equation modeling with AMOS: Basic concepts, applications, and programming. New York: Routledge.

Catino, M., \& Patriotta, G. (2013). Learning from errors: Cognition, emotions and safety culture in the $\begin{array}{llll}\text { Italian air force. Organization Studies, 34(4), 437-467. } & \text {. }\end{array}$ http://dx.doi.org/10.1177/0170840612467156

Cheng, B., Wang, M., Yang, S. J., \& Peng, J. (2011). Acceptance of competency-based workplace elearning systems: Effects of individual and peer learning support. Computers \& Education, 57(1), 1317-1333. http://dx.doi.org/10.1016/j.compedu.2011.01.018

Chiaburu, D. S., Dam, K. van, \& Hutchins, H. M. (2010). Social support in the workplace and training transfer: A longitudinal analysis. International Journal of Selection and Assessment, 18(2), $187-$ 200. http://dx.doi.org/10.1111/j.1468-2389.2010.00500.x

Choi, W., \& Jacobs, R. L. (2011). Influences of formal learning, personal learning orientation, and supportive learning environment on informal learning. Human Resource Development Quarterly, 22(3), 239-257. http://dx.doi.org/10.1002/hrdq.20078

Chuo, Y. H., Tsai, C. H., Lan, Y. L., \& Tsai, C. S. (2011). The effect of organizational support, self efficacy, and computer anxiety on the usage intention of e-learning system in hospital. African

RAC, Rio de Janeiro, v. 21, n. 5, art. 6, pp. 710-729, Setembro/Outubro, 2017, www.anpad.org.br/rac (oc) 
$\begin{array}{llll}\text { Journal of } & \text { Business } & \text { Management, } & 5(14),\end{array}$ http://dx.doi.org/10.4028/www.scientific.net/KEM.467-469.2137

Clarke, N. (2004). HRD and the challenges of assessing learning in the workplace. International Journal of Training and Development, 8(2), 140-156. http://dx.doi.org/10.1111/j.14682419.2004.00203.x

Coelho, F. A., Jr. (2004). Avaliação de treinamento à distância: Suporte à aprendizagem e impacto do treinamento no trabalho (Dissertação de mestrado). Instituto de Psicologia, Universidade de Brasília, Brasília, DF, Brasil.

Coelho, F. A., Jr., Abbad, G. D. S., \& Todeschini, K. C. D. L. (2005). Construção e validação de uma escala de suporte à aprendizagem no trabalho em uma instituição bancária brasileira. Revista Psicologia Organizações e Trabalho, 5(2), 167-196.

Coelho, F. A., Jr., Abbad, G. D. S., \& Vasconcelos, L. C. (2008). Análise da relação entre variáveis de clientela, suporte à aprendizagem e impacto de treinamento a distância. RAC-Eletrônica, 2(1), 88-104. Recuperado de http://www.anpad.org.br/periodicos/arq_pdf/a_687.pdf

Coelho, F. A., Jr., \& Mourão, L. (2011). Suporte à aprendizagem informal no trabalho: Uma proposta de articulação conceitual. Revista de Administração Mackenzie, 12(6), 224-253. http://dx.doi.org/10.1590/S1678-69712011000600010

Cohen, D. J. (1990). What motivates trainees? Training \& Development Journal, 44(11), 91-94.

Dawley, D. D., Andrews, M. C., \& Bucklew, N. S. (2008). Mentoring, supervisor support, and perceived organizational support: What matters most?. Leadership \& Organization Development Journal, 29(3), 235-247. http://dx.doi.org/10.1108/01437730810861290

Eisenberger, R., Huntington, R., Hutchison, S., \& Sowa, D. (1986). Perceived organizational support. Journal of Applied Psychology, 71(3), 500-507.

Ellinger, A. D. (2005). Contextual factors influencing informal learning in a workplace setting: The case of "reinventing itself company". Human Resource Development Quarterly, 16(3), 389-415. http://dx.doi.org/10.1002/hrdq. 1145

Figueirêdo, R. da S. (2008). Impacto do treinamento no trabalho: Análise de um curso técnicooperacional em uma organização do ramo de energia elétrica (Dissertação de mestrado). Instituto de Psicologia, Universidade Federal de Brasília, Brasília, DF, Brasil.

Hair, J. F., Black, W. C., Babin, B. J., Anderson, R. E., \& Tatham, R. L. (2009). Análise multivariada de dados. São Paulo: Bookman.

Hanke, C. (2006). Impacto do treinamento no trabalho: Análise de ações de capacitação de auditores do Banco do Brasil (Dissertação de mestrado). Universidade de Brasília, Instituto de Psicologia, Brasília, DF, Brasil.

Holton, E. F., III, \& Baldwin, T. T. (2003). Making transfer happen: An action perspective on learning transfer system. In E. F. Holtin \& T. T. Baldwin (Eds.), Improving learning transfer organizations (pp. 3-15). San Francisco: Jossey-Bass.

Holton, E. F., III, Bates, R. A., \& Ruona, W. E. (2000). Development of a generalized learning transfer system inventory. Human Resource Development Quarterly, 11(4), 333-360. http://dx.doi.org/10.1002/1532-1096(200024)11:4<333::AID-HRDQ2>3.0.CO;2-P

Jansen, J. J., Vera, D., \& Crossan, M. (2009). Strategic leadership for exploration and exploitation. The Leadership Quarterly, 20(1), 5-18. http://dx.doi.org/10.1016/J.LEAQUA.2008.11.008 
Kirwan, C., \& Birchall, D. (2006). Transfer of learning from management development programmes: Testing the Holton model. International Journal of Training and Development, 10(4), 252-268. http://dx.doi.org/ 10.1111/j.1468-2419.2006.00259.x

Kraimer, M. L., Seibert, S. E., Wayne, S. J., Liden, R. C., \& Bravo, J. (2011). Antecedents and outcomes of organizational support for development: The critical role of career opportunities. Journal of Applied Psychology, 96(3), 485-500. http://dx.doi.org/10.1037/a0021452

Lee, T., Fuller, A., Ashton, D., Butler, P., Felstead, A., Unwin, L., \& Walters, S. (2004). Workplace learning: Main themes perspectives. Learning as Work Research Paper, 1(2), 53-73.

Lohman, M. C. (2005). A survey of factors influencing the engagement of two professional groups in informal workplace learning activities. Human Resource Development Quarterly, 16(4), 501-527. http://dx.doi.org/10.1002/hrdq.1153

Lohman, M. C. (2009). A survey of factors influencing the engagement of information technology professionals in informal learning activities. Information Technology, Learning, and Performance Journal, 25(1), 43-53.

March, J. G. (1991). Exploration e exploitation in organizational learning. Organizational Science, 2(1), 71-87. http://dx.doi.org/10.1287/orsc.2.1.71

Marsick, V., \& Watkins, K. (2003). Demonstrating the value of an organization's learning culture: The dimensions of the learning organization questionnaire. Advances in Developing Human Resources, 5(2), 132-151. http://dx.doi.org/10.1177/1523422303251341

Mortimer, E. F., \& Scott, P. (2002). Atividade discursiva nas salas de aula de ciências: Uma ferramenta sociocultural. Investigações em Ensino de Ciências, 7(3), 283-306.

Pantoja, M. J., \& Borges-Andrade, J. (2004). Contribuições teóricas e metodológicas da abordagem multinível para o estudo da aprendizagem e sua transferência nas organizações. Revista de Administração Contemporânea, 8(4), 115-138. https://dx.doi.org/10.1590/S141565552004000400007

Pereira, L. M. R., Loiola, E., \& Gondim, S. M. G. (2016). Aprendizagem de competências, suporte à transferência de aprendizagem e desempenho docente: Evidências de validação de escala e teste de relações. Organizações \& Sociedade, 23(78), 438-459. http://dx.doi.org/10.1590/198492307856

Perrot, S., Bauer, T. N., Abonneau, D., Campoy, E., Erdogan, B., \& Liden, R. C. (2014). Organizational socialization tactics and newcomer adjustment moderating role perceived organizational support. $\begin{array}{llll}\text { Group } \quad \& \quad \text { Organization } & \text { Management, } & 39(3), & \text { 247-279. }\end{array}$ http://dx.doi.org/10.1177/1059601114535469

Ragins, B. R., \& McFarlin, D. B. (1990). Perceptions of mentor roles in cross-gender mentoring relationships. Journal of Vocational Behavior, 37(3), 321-339. http://dx.doi.org/10.1016/00018791(90)90048-7

Salehzadeh, R., Asadi, A., Pool, J. K., Ansari, M. R., \& Haroni, A. (2014). The influence of perceived organizational support on dimensions of learning organization: An empirical study of SMEs in Iran. The Learning Organization, 21(3), 206-219. http://dx.doi.org/10.1108/TLO-08-2013-0040

Tamayo, N., \& Abbad, G. S. (2006). Autoconceito profissional e suporte à transferência e impacto do treinamento no trabalho. Revista de Administração Contemporânea. 10(3), 9-28. http://dx.doi.org/10.1590/S1415-65552006000300002

Tracey, J. B., \& Tews, M. J. (2005). Construct validity of a general training climate scale. Organizational Research Methods, 8(4), 353-374. http://dx.doi.org/10.1177/1094428105280055

RAC, Rio de Janeiro, v. 21, n. 5, art. 6, pp. 710-729, Setembro/Outubro, 2017, www.anpad.org.br/rac 
Veloso, A. L. O. M., Silva, M. J., Silva, I., \& Caetano, A. (2015). Fatores que afetam a transferência da aprendizagem para o local de trabalho. Revista de Administração de Empresas, 55(2), 22-35. http://dx.doi.org/10.1590/S0034-759020150208

Vygotsky, L. S. (1984). A formação social da mente (J. Cipolla Neto, L. S. M. Barreto, \& S. C. Afeche, Trad.). São Paulo: Martins Fontes. (Obra original publicada em 1935)

\section{Dados dos Autores}

Bruno Chaves Correia-Lima

Av. Miguel Calmon s/n, 41830-001, Salvador, BA, Brasil. E-mail: brunoccl@hotmail.com

Elisabeth Loiola

Av. Miguel Calmon s/n, 41830-001, Salvador, BA, Brasil. E-mail: beteloiola10@gmail.com

Cícero Roberto Pereira

Cidade Universitária, s/n, 58051-900, Castelo Branco, João Pessoa, PB, Brasil. E-mail: crp@cchla.ufpb.br

Sônia Maria Guedes Gondim

Av. Miguel Calmon s/n, 41830-001, Salvador, BA, Brasil. E-mail: sggondim@gmail.com 